\title{
Cuba and the Global Year of Universal Health: Cristian Morales PhD Former PAHO/WHO Representative in Cuba
}

\author{
Gail A. Reed MS
}

A 2014 PAHO resolution that Cristian Morales helped formulate serves as a framework for the Americas' governments to actively work towards health for all their people, incorporating this aim into national programs for sustainable development. This September, the UN General Assembly will sponsor a High-Level Meeting on Universal Health . . . a first in its history. In part two of MEDICC Review's interview with Dr Morales, he outlines strategies he believes vital for transforming health systems to reach universal health-defined as coverage and access for all-and for turning words into action.

\begin{abstract}
MEDICC Review: The 2019 UN meeting comes on the heels of the 40th anniversary of the Alma Ata Declaration, which committed the world's governments to health for all. What are your expectations of the meeting and Cuba's possible contribution?
\end{abstract}

Cristian Morales: Alma Ata was a milestone in global public health, consolidating primary health care as fundamental, not only to achieve health for all but also to ensure the right to health the world over. For various reasons, Alma Ata's goals weren't fulfilled, falling short of its slogan Health for All by the Year 2000. Many important advances were made in the 80s and 90s, expanding health services for those who had gone without. But many times, these were limited to "basic packages" of care for the poor. And as the founder of the British health system said: policies for the poor are poor policies. That is, policies must leave no one behind and address the needs of the most vulnerable, but they have to be intended for everyone, universal. And this is the essence of Alma Ata that we're trying to recover 40 years later, that universal health be our guide for moving forward over the next decades. This is the vision that will enable us to implement the right to health and build health systems that fight inequities, are more efficient and express solidarity.

But now we face the huge challenge of going beyond words. Declarations are important as frameworks representing political consensus. But when you're faced with the reality of changing priorities-because we're also saying that health must have a higher priority - this can often clash with other interests. In any case, the idea is to find ways to implement the essential elements of Alma Ata in the workings of our health systems over the next decades, leading to more efficiency, improved health and wellbeing, and also more equitable and productive societies capable of generating more economic growth. And we have to be very clear on this: health is integral to economic growth, key to decent employment and to developing the potential of entire populations. You also have to recall that the majority of people working in health are women and in general these jobs are better than those in some other sectors, so prioritizing health also helps us struggle against gender-based inequities.

I believe the Alma Ata anniversary allows us to project a consensus for the 2019 UN General Assembly, leading to highlevel commitment by countries to placing health at the core of sustainable development as we move towards the year 2030. It gives us the chance to revitalize what we already know is needed: first and foremost a focus on health preservation and promotion. But we shouldn't stop there. We have to be capable of providing curative care at the primary and other levels, as well as palliative care and rehabilitation as needed, in other words integrated services. And these must be provided through an organizational structure inserted in communities that can include hospitals but isn't solely concentrated there.

MEDICC Review: This change in the model of carefrom hospital centered to people, family and community centered-was one of the four key strategies you outlined in our earlier conversation as essential to paving the way towards universal health. And the other three?

Cristian Morales: I should also note that this first strategy coincides with transformations in Cuba's own health system 
over time. The second strategy I would identify is health system governance: in most countries in our region, health systems are fragmented, meaning patients have to go to specific places for specialized care and continuum of care is broken. For example, if I have both diabetes and hypertension, I'll probably need to see two different specialists-specialists who rarely even talk to one another.

Care is also segmented by job and socioeconomic status. If I don't have the resources of the person next to me, we're going to be seen in different facilities, in different subsystems with different resources available to us. And that generates avoidable inequalities, what we term inequities.

Structuring consolidated governance that guides actors in the same direction with clear objectives-which lead to improved health and wellbeing, with fewer disparities in health and thus fewer inequities in the society at large-is a second requisite for promoting universal health. In Cuba, we see strong governance through the Ministry of Public Health, so we don't find the problems of fragmentation and segmentation we see in other countries.

The third strategy is the responsibility of the state for health. This has to do primarily with health financing. It's important to understand it this way because health systems often include a myriad of providers, both public and private (whether profitmaking or nonprofit such as those offered by some NGOs). We have to attract and work with all those who provide health services, realizing at the same time that the gaps are enormous in services and their quality.

So when we talk about the state's responsibility, we're first referring to its responsibility to ensure that all these systems and providers use the same protocols, act according to the same strategies, have the same standards. But also, and this leads us to the third strategy, that financing should come mainly from public funds. This is very explicit: it requires that the region's countries should spend public funds on health equivalent to at least $6 \%$ of the country's GDP. The reality is that very few countries in our region meet this goal: Cuba (which spends over 6\%), Canada, Costa Rica and one or two more.

The average is only $3.8 \%$, so when we are aiming at $6 \%$, this implies a significant shift in priorities for state spending. But it's quite necessary, since what we've witnessed empirically in the most developed (OECD) countries is that they may be capitalist, but they have socialized their health systems, mainly aiming at greater efficiency in use of resources but also stemming from a long-term commitment to their societies' development.

These OECD countries spend an average of $8 \%$ of GDP on public funding for health. So if we return to the $6 \%$ goal in our region, it's clear that this change would put us within reach of universal health. Why do I think so? Because it's not about undoing what's been done, but rather transforming what exists. And that is much easier when you have more resources. This doesn't mean taking away resources from one place to give to another: it means injecting more resources into health systems in order to respond to population health needs, but also to contribute towards liberating human potential that in turn drives development of the economy and society as a whole.
In essence, this is a win -win investment. And such an investment is backed by the experience of other countries' advances along this path.

\section{MEDICC Review: So, where to begin this transformation towards universal health?}

Cristian Morales: To begin such a process of transformation, which is bound to be different in each country, it might make sense to start by adopting one or another of the strategies first. But eventually, they have to dovetail, guided by a uniting vision. And that leads us to the fourth strategy: intersectoral action, because of course health doesn't depend on health systems alone.

In fact, studies show that social and environmental determinants carry more weight than health systems when it comes to population health results. This includes, for example, the interaction between health and education, health and agriculture, health and efforts to control vectors responsible for Zika and other viruses as well as to decrease antibiotic resistance. Early education to teach healthy habits in school children will serve population health throughout their generation. Intersectoral action with the economy can lead to healthier populations, with a social climate more conducive to ventures that generate better ideas and in which people are more productive and take more initiative. Another example is the environment, where through greater intersectoral action, the effects of climate change can be mitigated, protecting people from the risks it poses.

Taken together, these four strategies-a people-centered model of care, strong governance, public funding at least 6\% of GDP and greater intersectoral action related to social and environmental determinants-coincide with the reality of Cuba's health system, and of others as well. Yet, this is not a goal to be pursued by one country or even a group of countries: I believe our whole region of the Americas can advance towards universal health.

\section{MEDICC Review: In relation to what health systems need to be strong, to be able to meet the needs of their people, how do you see the changes in Cuba over the years?}

Cristian Morales: Health systems rely on a few essential elements to consolidate and offer quality services, promote health and prevent disease. First of course, they need human resources, and there is no doubt that Cuba has these as a result of a decision taken very early in the process of establishing a single public health system, a visionary decision. And that visionary decision was followed by another: creation of the health research institutes and during the very difficult economic situation in the 1990s, investments in the biopharmaceutical industry, biotechnology.

So, in addition to human resources, you need the kind of technology that comes from these institutions' accumulated knowledge transformed into medicines, new molecules, new procedures and equipment.

And of course you need financial resources as part of the equation, and also infrastructure. When, for various reasons, you have limited access to funding - as is the case in Cubathen it's very important to be able to bring in the hard currency 
to buy raw materials, for example, to produce the medications needed in the country. (Two thirds of the medications on Cuba's essential medicines list are produced domestically.) No matter the facilities and human resources that exist in Cuba to maintain this production, certain raw materials and active ingredients have to be bought abroad in hard currency. And add to that the third of medications that must be imported, including some of the latest, quite expensive technologies that Cuba doesn't yet produce that help prolong lives and improve quality of life. Making these technologies available is a responsibility that the health system has assumed, despite the cost.

MEDICC Review: Let's talk a bit more about Cuban biotech. It's been in the news because of the Cuban lung cancer therapeutic vaccine now in clinical trials in the USA by virtue of agreements with the Roswell Park Comprehensive Cancer Center in New York. And the number of novel products emerging from Cuba's biotech industry is surprising, given the size of the country. How do you see biotech's role in Cuba and internationally?

Cristian Morales: Biotech in general has been one of the great drivers for improving quality of life and extending life expectancy. The rhythm of transformative discoveries in health technologies is truly impressive, and in many countries this has created a paradox: even when these discoveries are made available to health systems, they are not always available to the people who need them.

The fact is that the biotech industry in most of the world is structured mainly to reap economic benefits. What's interesting in Cuba's case is that we see a high-quality industry with novel, innovative products like the lung cancer vaccine CIMAvax-EGF, others such as Heberprot-P for treating diabetic foot ulcers, and many more. The difference is that here we have an interconnected industry that from the moment research is initiated to produce new knowledge, forethought has already been given to how to transform that knowledge into technology, and how to integrate both into the entire health system. So that's another important experience Cuba has to offer, which also includes strict protocols for product validation through clinical trials.

Cuba's biotech industry's first mission is to place all its products and technologies at the service of the national health system. But at the same time, its products are marketed globally, benefiting the health of millions while generating revenues vital to sustaining a universal public health system like Cuba's.

MEDICC Review: Whatever the advances, all health systems face challenges. What are the main ones you see in Cuba?

Cristian Morales: In Cuba's case, I think the first big challenge is to maintain its achievements and plan strategies to reach higher

in the coming years. This is clearly recognized by the country's health leaders when they affirm, for example, the need to further improve mortality indicators in maternal-child health. One of the goals of the Ministry of Public Health is to reduce infant mortality to under 4 per 1000 live births, not an easy task.

Another great challenge for a health system like Cuba's is to make more efficient use of resources to guarantee its sustainability. They have already resolved efficiency at the macro level in two ways - first by having a single health system, which is more efficient than a fragmented one with different subsystems. This allows for more integrated, comprehensive patient care, including prevention. The second, which we've already mentioned, is that they provide public financing for the system.

However, many other sources of efficiency still have to be considered that can be improved in any system, including Cuba's. These have to do with planning and with purchase and distribution of inputs, resources, medications and so on. Reducing waste is another challenge for every health system: sometimes waste results from errors, other times it's due to loss of medicines because of occasional supply disruption, and of course like everywhere else, there are issues of corruption. No country is exempt from that.

So, several sources of efficiency can still be tapped to guarantee the health system's sustainability. If you look at the structure and organization of services: this is still guided by the public health law of 1983, when the social, economic, epidemiologic and demographic situation in Cuba was substantially different than today. Currently, Cuba's is one of the world's most rapidly aging populations: over $20 \%$ of its people are 60 years or older. This automatically presents quite a different epidemiologic picture, in which noncommunicable chronic diseases are the main causes of death and morbidity, and are also combined with old and new communicable disease threats (the old ones like dengue, which has been in Cuba for some years, and new ones such as Zika, chikungunya and other latent viruses). 
This challenge, then, is related to some outdated assumptions in the health system's legal framework, a challenge that if met can also produce greater efficiency in terms of better organization of patient services today. And finally, there's the challenge, at least in my view, of continuing Cuba's tradition of solidarity.

\section{MEDICC Review: Does PAHO/WHO play a role in addressing these challenges?}

Cristian Morales: Yes, I think so. As we analyzed these challenges, we conceived a strategy of "accompaniment", agreed upon with the Ministry of Public Health, around five main priorities. The first is more health, that is collaboration to help achieve better health outcomes; then comes more efficiency, followed by more intersectoral action (related to the sustainable development goals and explicitly how other sectors can promote health), more resiliency (such as mitigation of the effects of climate change), and finally more Cuba in global health.

These are the priorities that will guide our cooperation through 2022, expressed in an agreement signed in April 2018 at the CubaSalud Convention, where we had the privilege of signing the document with the WHO Director General, PAHO Director and Cuba's Minister of Public Health. (See https://apps.who .int/iris/bitstream/handle/10665/275329/ccs-cub-2018-2022-spa .pdf?ua=1-Eds.)

I think that PAHO and WHO can contribute a great deal to Cuba and other countries. I think our main goal right now is to help countries implement universal health and to facilitate collaboration among them.

MEDICC Review: In this year when the goal of universal health is once again on the global agenda, from your experience, what do you see as Cuba's contribution to these debates? What does Cuba have to offer, even in dialogue with countries that don't share its social or economic system?

Cristian Morales: I think Cuba's most important contribution is proving that it can be done...universal health. Cuba has shown that even with few resources, if there is determination, you can achieve health results beyond what might be expected of a small, resourcelimited country facing a difficult geopolitical context. It's been complex for Cuba since its revolution. But despite this, they've done more than many countries with greater resources, and not only in health outcomes and quality of life for the Cuban population. They have managed to build bridges between people, between countries. -1 - 